Cordula Reichart

\title{
Exemplum sublimierter Leidenschaft oder Modell einer «natürlichen» Gemeinschaft?
}

\author{
Zu Begriff und Funktion der Natur in Pierre Abélards Historia \\ calamitatum und der Correspondance mit Héloïse
}

DOI 10.1515/zrp-2016-0030

\begin{abstract}
The following article investigates how Abélard functionalises concepts of nature as part of the philosophical and literary discourse in his Historia Calamitatum and correspondence with Héloïse. Nature and nature metaphors in the literary work of the Letters prove to be more than simply a means for reflecting upon and imagining that which, due to dogmatic and generic constraints, encountered enduring resistance in philosophy and theology. They offer a glimpse of Abélard as a great reformer by providing insights into a new monastic order that, as will be demonstrated by comparison with the Aristotelian concept of friendship and by contrast with Giorgio Agamben's The Kingdom and the Glory: For a Theological Genealogy of Economy and Government (Homo Sacer II, 2), questions contemporary mediaeval economic relations. The Abélardian bond of love in Christo, the new monastic communitas of Paraclete, is constructed as the model of what can be called a more «natural» community, a model which here for the first time receives thorough systematic analysis.
\end{abstract}

Keywords: Pierre Abélard, definitions and conceptualisations of nature, natural law, Scholasticism, rhetoric and philosophy of the 11th and 12th centuries in France, dogmatic and generic constraints, Aristotelian concept of friendship, economy and community

Schlagwörter: Pierre Abélard, Naturbegriffe und -konzeptionen, Naturrecht, Scholastik, Rhetorik und Philosophie des 11. und 12. Jahrhunderts in Frankreich, Disziplinäre und institutionelle Zwänge, aristotelisches Freundschaftskonzept, Ökonomie und Gemeinschaft

Korrespondenzadresse: Dr. Cordula Reichart, Ludwig-Maximilians-Universität München, Institut für Romanische Philologie, Schellingstr. 3, 80799 München, E-Mail: cordula.reichart@romanistik.uni-muenchen.de 


\section{Einleitung}

Im Werk Pierre Abélards nimmt der sogenannte Paraklet-Briefwechsel mit Héloïse eine Sonderstellung ein. Er stellt, als ein literarisches Zeugnis, seine einzige nicht ausschließlich philosophische oder theologische Schrift dar. Zudem setzt er sich aus drei sehr unterschiedlichen Teilen zusammen, in die verschiedene literarische Gattungen eingehen: die als Trostbrief an einen fiktiven Freund vorangestellte Lebens- und Leidensgeschichte Abélards, der eigentliche, konsolatorische Briefwechsel zwischen Abélard und Hélö̈se und schließlich die Klostergründungsdokumente mit Nonnenspiegel und jenen von Abélard für Héloïses Nonnen zusammengestellten Klosterregeln. Diese «Verschränkung der literarischen Genera», ${ }^{1}$ der allgegenwärtige stilistische «Doppelsinn» und die differenzierte kompositorische Einheit haben in der Forschung zu Authentizitäts- und Autorschaftsproblemen geführt, die von der Frage geleitet waren, ob die Texte eine Einheit bilden und von wem der Briefwechsel sei, Abélard und/oder Héloïse, oder, ob er gar eine nachträgliche Fälschung sei. ${ }^{2}$ Trotz nach wie vor gebotener Vorsicht ${ }^{3}$ gehen die meisten Autoren mittlerweile von der Echtheit der Schriften aus, die in der Regel als Ausdruck dessen gelesen werden, was sie am offensichtlichsten exponieren: das Liebesverhältnis von Abélard und Héloïse einerseits, das der Briefwechsel zur geistigen Beziehung der beiden konvertiert, die Gründung des Reformklosters Paraklet (das heißt 'Tröster') zur Zeit der großen Klosterreformen andererseits.

Ungebrochen stößt die mittelalterliche Verhandlung der zwischenmenschlichen Liebesbeziehung auf größtes Interesse. Sehr viel weniger häufig rücken in den Paraklet-Briefen dagegen die Metaphorik und die disparaten Konzepte der Natur in den Vordergrund, die dem Leser dabei allenthalben begegnen. Dies verwundert insofern, als diese zeitgleich in der Philosophie des elften und zwölften Jahrhunderts, insbesondere in der sich etablierenden Scholastik, eine bedeutende Rolle spielen und Abélard durch sein Studium bei Anselm von Laon mit einem führenden Theoretiker des Naturrechts, der lex naturae, sowie Fragen der Vermittlung und theologisch-philosophischen Verhandlung schöpfungsrelevanter, antiker und christlicher Naturkonzepte in Berührung gekommen ist. ${ }^{4}$ Gerade

1 Cf. das vorzüglich kommentierende Nachwort von Krautz zu Abaelard (1989, 381), zur Differenziertheit der Textstruktur und Gattungen auch von Graevenitz (1992, insbesondere 31).

2 Cf. eines der Grundlagenwerke der Abélard- und Héloïse-Forschung: Moos (1974) sowie Schmeidler (1935), im Anschluss an Schmeidler, Benton/Prosperetti Ercoli (1975).

3 Cf. Moos (1997, insbesondere 36s.) sowie Flasch (2000, 236-252).

$4 \mathrm{Zu}$ Abélards Theologiestudium bei Anselm von Laon, das auf das Jahr 1113 datiert wird, z.B. Krautz (Abaelard 1989, 363). Die lex naturae war bei Wilhelm von Champeaux und der gesamten 
die abstrakten Naturkonzepte, aber auch empirische Naturphänomene, die im elften und zwölften Jahrhundert zunehmend an (wissenschaftlicher) Beachtung gewinnen, ${ }^{5}$ erweisen sich als besonders aufschlussreich, nicht nur für die «Natur» des Menschen, den Umgang mit den Leidenschaften und das Verhältnis der Liebenden, das hier im Vordergrund steht. Sie geben auch Auskunft über die intellektuelle Entwicklung Abélards, die Genese seiner methodischen Stoßrichtungen und die Beziehung zu den ordnungsstiftenden frühscholastischen Normen in einer Zeit des gesellschaftlichen Wandels sowie der institutionellen und disziplinären Zwänge. ${ }^{6}$

Den gesamten Briefwechsel hindurch rekurriert Abélard - und gelegentlich auch Héloïse - auf die Semantik und Konzepte der Natur, auf ihre tradierten Muster und Metaphern. Wie ein basso continuo grundieren sie den Briefwechsel. Mit der stark rhetorisierten Naturmetaphorik verbindet sich indes nicht nur ein motivgeschichtlich aussagekräftiges Anliegen. Untersucht man die Verwendung der Naturmetaphorik und Naturkonzepte in den Paraklet-Briefen strukturell, so zeigt sich zunächst, dass sie als Exempla - auch im scholastischen Sinne des Wortes - oder als Analogien verwendet werden, dann aber, dass sie eine Art Wesenskern darstellen und ganz gezielt hermeneutisch elaboriert und funktionalisiert sind. Der folgende Beitrag fragt daher am Beispiel der Historia Calamitatum und des Briefwechsels nach der Funktion der Naturkonzepte im philosophischliterarischen Diskurs Abélards: Auf welche Konzepte und Metaphern rekurriert er, wie wird der Naturbegriff rhetorisch-argumentativ eingesetzt - und $\mathrm{zu}$ welchem Zweck?

Die Natur- und Pflanzenmetaphorik soll im Folgenden als Reflexionsfigur angesehen werden, durch die sich in den ästhetischen Schriften des Briefwechsels denken lässt, was in Philosophie und Theologie aus disziplinären und dogmatischen Gründen auf permanenten Widerstand stößt bzw. (noch) keinen institutionalisierten Ort der systematischen Reflexion hat: Es wundert daher nicht, dass ausgerechnet in der Modellierung der Natur die rhetorische Artifizialität und Rhetorizität zum Ausdruck kommen. Die zahlreichen Naturmetaphern und ver-

Schule von Laon Gegenstand der Auseinandersetzung mit den Erzvätern und den Schriften des Hl. Augustinus sowie Thomas von Aquins. Einen ausführlichen Überblick zur Natur im Denken und den wissenschaftlichen Disziplinen des Mittelalters vermittelt der Doppelband von Zimmermann/Speer (1992).

5 Wegmann (2005) weist nach, wie insbesondere in den Klöstern und Klosterhandschriften ab dem 11. Jahrhundert ein Interesse an Naturbeobachtung auszumachen und ein qualitativer und quantitativer Wandel in der Naturwahrnehmung festzustellen ist.

6 Abélard schreibt in einer Umbruchsphase, cf. Flasch (2000, 236-240), Flasch sieht daher in Abélard eine «Schlüsselfigur des 12. Jahrhunderts [...] an ihm traten die Widersprüche seines Jahrhunderts zutage» (ib., 236). 
schiedenen Naturkonzepte sind Teil der rhetorischen Technik und der argumentativen wie logischen Progression. Mehr noch. Sie unterliegen einem Prinzip. Indem an ihnen Fragen der Ordnung verhandelt werden, verweisen sie auf eine für das Mittelalter wesentliche, aristotelische Stoßrichtung. ${ }^{7}$ An ihnen entspinnt sich daher regelrecht eine eigene Argumentation: Die Naturreferenzen erweisen sich als eine textuelle Strategie, die systematisch, methodisch, eingesetzt, und bisweilen wohldurchdacht in die Nähe des Bruderkriegs bzw., umgekehrt, des antiken amicitia-Konzepts gerückt, für reformatorische Zwecke funktionalisiert wird. Die von Abélard als brudermörderisch dargestellten Klostergemeinschaften sollen hier deshalb als Folie betrachtet werden, vor deren Hintergrund sich ein anderes Konzept des Gemeinwesens konturieren lässt, das nicht nur als Gründung des Klosters Paraklet, sondern als alternatives communitas-Modell in den Blick zu rücken ist. Kurz, anhand einer Naturmetaphorik, die in der Argumentation durch Art, Auswahl und intertextuelle Bezüge auf strategische Weise zum Tragen kommt, soll in dem Briefwechsel ein doppeltes Reformanliegen verdeutlicht werden: einer Überdisziplinierung auf der Seite der dogmatischen Disziplinenlehre der mittelalterlichen Philosophie und Theologie und einer A- bzw. De-Regulierung auf Seiten der ökonomischen Moral und sozialen Ordnung der zeitgenössischen Klostergemeinschaften. Gegen sie setzt Abélard - unter Rekurs auf das aristotelische und scholastische amicitia-Konzept - ein in systematischer Hinsicht wenig beachtetes Modell einer «natürlichen» communitas: Das berühmte Exemplum sublimierter Leidenschaft ist in diesem Sinn also als Modell einer anderen, «natürlichen», von innen heraus zu ordnenden Gemeinschaft zu betrachten.

\section{Exemplarische Abgrenzung zum philosophischen und theologischen Werk? Naturbegriff und Pflanzenmetaphorik in der Historia Calamitatum}

17 Jahre nach dem Liebesverhältnis mit Abélard stößt Héloïse auf die Historia Calamitatum und reagiert in Form eines ersten (in der üblichen Wiedergabe des Briefwechsels, des zweiten) Briefs. In dieser «Histoire des Malheurs», der Lebensund Leidensgeschichte Abélards, wird nicht nur die Abgrenzung zu Abélards

7 Grundlegend zum ordo-Gedanken in der Philosophie des Mittelalters, vor allem am Beispiel Thomas von Aquins, Krings (1982). 
anderen, theoretischen Schriften besonders deutlich. ${ }^{8}$ Ihr kommt für die Konzeption und Erwähnung der Naturbegriffe eine Schlüsselrolle zu.

Doch nicht in der Historia Calamitatum und dem Briefwechsel ist die Forschung Begriff und Konzept der Natur nachgegangen, sondern in Abélards philosophischen und moraltheologischen Hauptwerken: zum Beispiel in seinem wenig später erscheinenden ethisch-moralischen Hauptwerk, der sogenannten Ethica seu scito se ipsum (Ethica oder Erkenne dich selbst), deren wesentliche Aspekte Abélard in der autobiografischen Wiedergabe seines Lebens und seiner Schwächen bis einschließlich der Klostergründung und der drohenden Todesgefahr aus der Perspektive des vernünftig Reflektierenden bereits vorausnimmt. ${ }^{9}$ In dieser scholastisch-moraltheologischen Schrift stellt Abélard die Vernunft als Prinzip menschlichen Handelns dar, Verpflichtung (obligatio) und Gesetz (lex) werden vor dem Hintergrund des mittelalterlichen «Naturrechtsdenkens» zunehmend begrifflich verknüpft. ${ }^{10}$ Der Abélard-Spezialist Jean Jolivet kommt bei seiner Untersuchung der Naturbegriffe bei Abélard zu dem Ergebnis, dass dieser sich kontinuierlich, indes eklektisch und heterogen auf die zeitgenössisch diskutierten Naturkonzepte beziehe: ${ }^{11}$ Während die Natur für den Logiker Abélard ein notwendiges «Referenzkonzept» darstelle, auf das er sich «abstrakt» bezieht, bleibe es in moralischer Hinsicht als "corruptio naturae» sehr vage. ${ }^{12}$ Einen gänzlich neuen Aspekt fügt er jedoch, so John Marenbon, dem oben erwähnten Naturrechtsgedanken, der sogenannten lex naturae zu, den er im Anschluss an Paulus' Römerbrief und Augustinus' Deutung desselbigen in seinen Hauptwerken der Ethica und der Theologia Christiana neuinterpretiert und auch in die Briefe, insbesondere in den VII. Brief, einfließen lässt. ${ }^{13}$ Marenbon verweist darauf, dass Abélard sich mit seiner Auslegung des Römerbriefs und der aus der Urzeit der

8 Im Folgenden beziehe ich mich auf die Textausgabe Abélard et Héloïse (2000), cf. für das Zitat (Abélard et Héloïse 2000, 57). Diese Ausgabe druckt die Briefdokumente bis einschließlich des achten Briefs, die Ausführungen zur Klosterökonomie und die Gründungsdokumente finden sich in der Ausgabe Abaelard (1989).

9 Cf. Abaelard (2006), auch hier spielt die Liebe eine zentrale Rolle, cf. beispielsweise Perkams (2001).

10 Hartung $(1999,21 s$.$) zeichnet einleitend die Geschichte der obligatio und ihre Formierung im$ Mittelalter unter Einfluss von Thomas von Aquin und der Auslegung von Paulus' Römerbrief nach und zeigt, wie das «theologische Element» der Verschuldigung des Menschen und das «rechtspolitische Element der Verpflichtung» (obligatio) zusammenfinden und - unter Vermittlung von Francisco Suàrez, Hugo Grotius u. a. - noch in die frühaufklärerischen Naturrechtslehren eingehen.

11 Jolivet (1966, 297-304).

12 Jolivet (1966, 303s. [Übersetzung C. R.]).

13 Cf. v.a. Marenbon (1992, insbesondere 618-621) sowie id. (1997). 
Naturrechts überbrachten «Goldenen Regel» (die die Schule von Laon der philosophischen und moraltheologischen Auslegung der lex naturae als Basis zugrunde legt) gegen seinen ehemaligen Lehrer Anselm von Laon und seine Schüler wende: Abélard nämlich differenziere im Anschluss an die antike Philosophie (Platons und seiner Exegeten) als erster zwischen einer kosmischen lex naturae und der (gesellschaftlichen Applikation als) ius positiva, zudem nutze er seinen philosophischen Dialog (die Collationes) gezielt, um neben der theologischen Position eine philosophische $\mathrm{zu}$ artikulieren, die anderswo keinen «Ort» habe. ${ }^{14}$

Daran lässt sich anknüpfen, doch muss man dazu etwas ins Detail der ästhetischen Schriften gehen. Denn auch in der Historia Calamitatum und im Briefwechsel lässt sich eine eklektische Referenz auf überbrachte Naturkonzepte ausmachen. Allerdings, diese bleibt alles andere als vage. So taucht die Natur an zahlreichen Stellen des Briefwechsels und der Historia Calamitatum in kodierten Formen und mittelalterlich etablierten Konzepten auf, das heißt vor allem: als Zitate der antiken, spätantiken und christlichen Naturkonzeptionen. Doch Abélard übernimmt die überbrachten Muster nicht einfach. Er gibt ihnen eine ganz eigene erkenntnistheoretische Wendung: Die Naturreferenzen werden weniger oder, besser gesagt, nur indirekt für die Verhandlung der menschlichen Natur und der Grundsatzreflexion über die moralische Verantwortung des Individuums (das heißt für die philosophisch-moraltheologische Debatte) verwendet. Vielmehr dient die Metaphorik der Natur Abélard konsequent dazu, die eigene geistigphilosophische und institutionelle Entwicklung $\mathrm{zu}$ illustrieren, konkrete Fragestellungen seines Lebens mit theoretischen Problemhorizonten an der Schnittstelle von mittelalterlicher Theologie und Philosophie zu verbinden. Gerade mit Blick auf die Historia Calamitatum könnte man auch sagen, er lote in der ästhetischen Schrift Anwendungsbereiche der theoretischen Konzepte aus, das, was in der scholastischen Theorie keinen Platz der systematischen Reflexion hat.

Programmatisch begibt er sich dafür auf das Gebiet der Ästhetik und damit auf ein Feld, das per definitionem aus dem zeitgenössischen philosophischen Disziplinenkanon herausfällt. Das subtile Spiel mit Disziplinenfragen (gerade da, wo es offensichtlich um Disziplinierung anderer Art, die Beherrschung der Leidenschaften geht) macht gleich der erste Satz der Historia Calamitatum unmissverständlich deutlich: «Souvent l'exemple a plus d'effet que la parole pour exciter ou pour calmer les passions humaines» (Abélard et Héloïse 2000, 57). Gegenübergestellt werden «l'exemple» und «la parole», ein als eigenes Argument angeführtes autobiografisch-rhetorisches exemplum gegen die theoretischen Auslassungen. So unmissverständlich die Distanznahme dazu, dass es nun nicht primär um Phi-

14 Cf. Marenbon (1992, 614-618). 
losophietheorie geht, so ausdrücklich wird auch die Nähe zur Erzählung eines Beispiels, eines «récit vivant», der, ganz rhetorisches exemplum, die individuelle, autobiografische Illustration mit einer Reflexion auf eine praktische, exemplarisch-allgemeingültige Applikation verbindet. Der unablässig für das «Recht des denkenden Subjekts» ${ }^{15}$ kämpfende Abélard tritt hier nicht (nur) als Theologe, Logiker oder Ethiker auf, das heißt, in den etablierten (philosophischen) Disziplinen; er behauptet sein Recht auf dem Gebiet der Ästhetik und für die Gattung der Autobiografie bzw. des (autobiografischen) Briefwechsels eine eigene Erkenntnisfunktion. Wenn es als richtungweisend zu verstehen ist, dass der Wortlogiker und Dialektiker die eigene, angestammte Disziplin gleich zu Beginn des Textes verlässt, oder zumindest in ein dialektisches Verhältnis zu dem Sachgebiet der Rhetorik (als Grundlage der scholastisch-dialektischen Methode) versetzt, müsste dann nicht auch der Rekurs auf die Gattung der Autobiografie anders verstanden werden ${ }^{16}$ Nicht die immer wieder betonte starke Subjektposition, ${ }^{17}$ die Artikulation seines Selbstbewusstsein, ${ }^{18}$ nicht die Erneuerung mittelalterlichen autobiografischen Schreibens stünden im Vordergrund, ${ }^{19}$ sondern die Exponiertheit der Gattung Autobiografie in Fragen der Beispielhaftigkeit, der Verbindung von Theorie und Praxis, als Garant für einen «Sitz im Leben» und einer Reflexion auf Entwicklungen von Person und Zeit. Autobiografie also im Dienste der logischen Beweisführung, der Allgemeingültigkeit und (kritischen) Prüfung des eigenen und fremden Verhaltens, aber auch des exemplarischen, ökonomischen Ordnens und Verwaltens.

Ist diese gattungstechnische Schwerpunktverschiebung daher nicht als eine rhetorische Strategie der Übertragung zu begreifen, bei dem sich Austragungsort und Kampfplatz ändern? Die zu Beginn der Lebensgeschichte benannte und von der Forschung vielbeschworene «Turnier- und Gefechtkunst», der zufolge der Gelehrte Abélard, der biografisch auf das weltliche Rittertum verzichtet hat, «buchstäblich wie ein Ritter [...] in die Gefechte mit seinen Lehrern und mit seinen Widersachern [...]» ziehe, ${ }^{20}$ bekäme in diesem Zusammenhang eine weitere Sinn-

15 Cf. zu diesem Aspekt Krautz (Abaelard 1989, 378).

16 In gattungstheoretischer Hinsicht ist die Historia Calamitatum ein Text, der stark auf antiken und mittelalterlichen, theologischen wie philosophischen Gattungskonventionen aufruht, auf dem Trostbrief wie auf antiken und christlichen hagiografischen Formen und Augustinus' Confessiones. Eine Aufstellung hierzu bieten die meisten Einführungen ins Werk, so z. B. Krautz (Abaelard 1989, 381), theoretische Überlegungen zur textkonstituierenden Funktion der Gattungsvielfalt finden sich ib. sowie Asper $(2002,113)$.

17 Abélards starke Subjektposition geht dabei auch auf das zurück, was man mit seiner Methode verbindet, die sich systematisch als Intentionsethik bezeichnen lässt, cf. z. B. Moos (1997, 36-45).

18 Flasch $(2000,237)$.

19 So eine zentrale These des Aufsatzes von Graevenitz (1992, 25).

20 Cf. Graevenitz (1992, 32). 
dimension; denn in den biografischen Genera der Leidensgeschichte und des Briefwechsels kann eine Praxis reflektiert werden, die in Logik und Rhetorik keinen Platz hat, und eine Kombination verschiedener Theorien zur Anwendung kommen, die die dogmatische, scholastische Disziplinenlehre teilt. ${ }^{21}$ Avant la lettre, im doppelten Sinne des Wortes, nämlich vor dem eigentlichen Briefdialog und vor der offiziellen Etablierung der Ästhetik als philosophische (Fundamental-)Disziplin im 19. Jahrhundert in der Nachfolge Kants und der modernen Subjektgeschichte, entwirft, kann man vielleicht sagen, Abélard die Ästhetik als eine Disziplin mit einer eigenen Erkenntnisfunktion (dass sie der Logik gleichzustellen sei, wird sehr viel später im 18. Jahrhundert Alexander Gottlieb Baumgarten ausdrücklich fordern). ${ }^{22}$

Sieht man nun genauer auf Abélards Naturreferenzen, bemerkt man schnell, dass die Natur- und Pflanzenmetaphorik in der Historia Calamitatum als exempla und Analogien fungieren. Der Wortlogiker nutzt sie gezielt, und in einer für die mittelalterliche Rhetorik charakteristischen Weise, zur logischen Verstärkung der Argumente, aber auch zu ihrer (kritischen) Reflektion. Weil die Analogien zwar eine skeptische, hermeneutische, bisweilen hermetische Vieldeutigkeit implizieren, Abélard aber (noch) nicht grundlegend am Erkenntniswert der Sprache und Welt zweifelt, kann er sie zugleich als «Schattenrisse der Wahrheit» bezeichnen. ${ }^{23}$ Wie sehr in der Natur aber ein künstlicher ordo waltet, erkennt man dann, wenn man das Prinzip ernst nimmt, das sich mit diesem verbinden und in den theologischen Problem- und damals offenen, aristotelischen Fragehorizont der «Trinität» Ökonomie, Ordnung, Regierung stellen lässt. Diese von Aristoteles der mittelalterlichen Scholastik hinterlassene theologisch-ökonomische Frage nach (der Natur) einer (göttlichen) Einheit der gubernatio ist einer der Ausgangspunkte der «Geschichte der ökonomischen Theologie», die Giorgio Agamben in seiner Schrift Herrschaft und Herrlichkeit. Zur theologischen Genealogie von Ökonomie und Regierung ausgehend von Aristoteles Metaphysik in der christlichen Theologie nach-

21 Eigentlich trennt die mittelalterliche Philosophie noch nicht zwischen den Disziplinen, cf. Flasch (2000, 13ss.). Die dialektische Methode der Scholastik allerdings markiert ein klar umgrenztes Feld. Es verwundert daher nicht, dass sich hier, v. a. bei Thomas von Aquin, die ersten wissenschaftstheoretischen Ausformungen von Einzeldisziplinen artikulieren, Abélard kannte die zeitgenössischen Einteilungsversuche und -schemata, beides zeigt Achtner (2008, 98s., insbesondere die Fußnoten 127-129 mit weiteren Belegen).

22 Cf. Baumgarten (1983) sowie zur Theoriegeschichte der Ästhetik Campe/Menke/Haverkamp (2014).

$23 \mathrm{Zu}$ den sprachphilosophischen Hintergründen erteilt Auskunft, Krautz (Abaelard 1989, 379): die gehäufte Verwendung von Analogien wurzelt u.a. in Abélards Platon-Rezeption und dem Einfluss seines «Lehrmeisters», dem «Nominalisten und Ketzer Roscelin» (ib.). 
zeichnet. ${ }^{24}$ Verkürzt gesagt geht es Agamben, seinem genetischen Ansatz und dem Bruch, den er genealogisch zwischen mittelalterlicher trinitarischer «oikonomia» und moderner Ökonomie sieht, um eine klare (Bruch-)Linie. ${ }^{25}$ Sein Paradebeispiel ist daher Thomas von Aquin und sein aporetischer Ordnungsbegriff («taxis»). ${ }^{26}$ Nicht nur, dass Abélard mit seiner «taxis» eine Praxis verbindet. Was Abélards Position so interessant macht, ist, dass sich bei ihm ein Gemeinschaftsund Ordnungskonzept skizzieren lässt, das die damals rekurrenten Fragen der Ökonomie ebenfalls aufwirft, das diesen allerdings einen weiteren Aspekt hinzufügt und damit einer anderen Genealogie Aufmerksamkeit verschafft.

Abélards Argumentation verfährt dabei so, dass die Naturbegriffe beinahe ausschließlich in Zusammenhang mit den Meilensteinen und Wendepunkten seiner Lebensgeschichte auftreten: Die Naturmetaphorik fokussiert die Meilensteine in der Historia Calamitatum auf Abélards Erkenntnisdrang und Beruf(ung) hin und strukturiert diese genauestens, sogar derart, dass neben seiner intellektuellen Entwicklung, seinem «Naturtalent», etwa das Wertesystem markiert und kommentiert wird: Abélards Herkunft vom Land, im Text als «vertu de ma terre» bezeichnet, wird der «culture littéraire» (Abélard et Héloïse 2000, 57) gegenübergestellt, gegen diese - nach der Ankunft in Paris und mit der Wahl des besonders blühenden Studienfaches - regelrecht eingetauscht: «J'arrivai enfin à Paris, où depuis longtemps la dialectique était particulièrement florissante» (Abélard et Héloïse 2000, 58). Wenn die Naturbelege persönliche Problemfelder, innere Konflikte und kommende institutionelle Attacken ankündigen, dann sind immer auch die aristotelischen Stichworte präsent, die den zeitgenössischen theologischen Problemhorizont ausmachen: das Verhältnis des Partikularen zum Universellen, von (erster) Ursache und Wirkung, der Bezug zu einem wahren transzendenten Regime der Schöpfungsordnung, zum Kosmos, ein «ökonomisch-providentielles» Schema, die Ordnung des Hauses im Gegensatz zur Stadt. ${ }^{27}$ Die Logik des Textes verfährt dabei in doppeldeutigen Metaphern: Intertexte, die eigentlich dem Bereich des Liebestrostes entstammen, werden in soziale Kontexte eingerückt, wie beispielsweise das Ovid-Zitat aus den Remèdes d'amour: «La grandeur est en butte à l'envie: c'est contre les cimes élevées que se déchaînent les vents» (Abélard et Héloïse 2000, 61). Eine weitergehende Betrachtung bestätigt, dass Fragen des Liebesbundes mit Fragen der Gemeinschaft korrelieren und präzisiert ferner, dass Abélard Eingriffe in die natürliche oder bestehende Ordnung, ihre Verände-

24 Cf. hierzu die Ausführungen in Agamben (2010).

25 Agamben (2010, 14ss., 32ss., 332ss.).

26 Agamben (2010, 107ss.).

$27 \mathrm{Zu}$ diesen aristotelischen Kernbegriffen: Agamben (2010, 106ss.), zur Ordnung von Haus vs. Stadt, ib. (32). 
rung, Konversion oder gar Perversion insbesondere an der kosmischen Welt- und Schöpfungsordnung reflektiert: Eine solche kosmische Dimension erreicht die Naturmetaphorik mit der Geburt des Sohnes «Astrolabe» (Abélard et Héloïse 2000, 70). So verwundert es auch nicht, wenn Héloïse nach der Geburt ihres Sohnes auf den «ordre naturel» rekurriert, um Abélards Ehegesuch in der Bretagne zunächst ganz abzulehnen, dann aber einer geheimen Ehe zuzustimmen. An der vorsehenden Natur- und Schöpfungsordnung werden hier Fragen der Un-/ Vereinbarkeit von Beruf und Berufung und der institutionellen Verankerung verhandelt. Denn Abélard, so die mit dem «ordre naturel» verbundene Argumentation, wurde von der Natur für alle geschaffen und solle seinem Ruf und Dasein als Philosoph keinen Abbruch tun: «Quel acte indécent et lamentable, moi que la nature avait créé pour tous, de m'asservir à une seule femme et de me soumettre à une si grande honte!» (Abélard et Héloïse 2000, 71). Der «ordre naturel» der irdischen Ehe wird nach der Geburt des Sohnes umgedreht: dem Sohn aus Fleisch folgt «in geistiger Fruchtbarkeit» die Zeugung der Töchter im Geiste. ${ }^{28}$ Verabschiedet wird im Folgenden eine alte, die Liebenden, den Denker, die Geschlechter, die Natur/Kultur und die Gemeinschaft spaltende Ordnung, zugunsten einer neuen, der «nouvelle plantation» des Doppelklosters, das all das in einem Ganzen, genauer: in einem «Haus», zu vereinen scheint, was zuvor als gespalten präsentiert wurde.

Ich möchte daher die Aspekte von Abélards weiterem Werdegang herausgreifen, in denen der Rekurs auf die Naturkonzepte den engen Zusammenhang von subjektiver Erkenntnisleidenschaft, Behauptung der eigenen Methode und institutionellem Bruderkrieg verdeutlicht. Denn Abélard, der mit Hilfe der Natur die Brillanz der eigenen Methode herausstellt, ist zugleich derjenige, der damit die pikantesten institutionellen Bruderkriege provoziert. Markiert werden auf diese Weise die zeitgenössischen Gefechte auf dem Gebiet der philosophischen und theologischen Disziplinenlehre. Einiges spricht jedoch dafür, dass sie die Hintergrundfolie nicht nur für den Liebes-Bund in christo bilden, sondern gleichzeitig die ideale Kontrastfolie darstellen für die Installation eines anderen communitas-Konzepts, dafür, dass mit der offensichtlichen conversio der Liebe noch weitere einhergehen. Aber der Reihe nach.

28 Cf. Vinken $(2002,183)$. 


\section{Natur und Bruderkrieg: von der Behauptung der eigenen Methode zur institutionellen Neugründung}

Als exemplarisch für Abélards Behauptung der eigenen Methode kann der Vergleich seines Lehrers Anselm von Laon mit einem scheinbar prachtvollen, in der Tat aber sterilen Baum gelten:

«'allai donc entendre ce vénérable vieillard. C'était à la routine, il est vrai, plutôt qu'à l'intelligence et à la mémoire qu'il devait sa réputation. Allait-on frapper à sa porte et le consulter sur une question douteuse, on en revenait avec plus de doutes. Admirable aux yeux d'un auditoire, dans une entrevue de consultation il était nul. Il avait une merveilleuse facilité de parole, mais le fond était sans valeur et manquait de sens. Lorsqu'il allumait un feu, il remplissait la maison de fumée, mais ne l'éclairait pas. C'était un arbre tout en feuilles qui, de loin, présentait un aspect imposant: de près, et quand on l'examinait avec attention, on le trouvait stérile. Je m'en étais approché pour recueillir du fruit; je reconnus que c’était le figuier maudit par le Seigneur, ou le vieux chêne auquel Lucain compare Pompée dans ces vers: «Ce n'est plus que l'ombre d'un grand nom: tel le chêne altier dans une campagne fécondè.

La chose reconnue, je ne demeurai pas longtemps oisif sous son ombre» (Abélard et Héloïse 2000, 62).

In diesem Textausschnitt korreliert Abélard zentrale Elemente aus Genesis 3, das Ergreifen der verbotenen Frucht vom Baum der Erkenntnis, mit einem Verweis auf Lucans alte, morsche Eiche aus dessen Bürgerkriegsepos Pharsalia. ${ }^{29}$ In der Lucan'schen wie der augustinischen Intertextualität verleiht Abélard, wie die Forschungsliteratur bereits unter verschiedenen Gesichtspunkten herausgehoben hat, der brüdermörderischen Kloster- und Geistesgemeinschaft Ausdruck. ${ }^{30}$ Mehr noch. Während die Eiche Lucan als Vergleich für Pompeius dient, der vom geringsten Lufthauch umgeweht werden könnte und erst recht vom Blitz des Gegners Caesar, gelingt Abélard mit dieser Zitatkombination ein doppelter Schlag:

Erstens nähert er sich hier dem Lehrer, in der durch den traditionellen Baumvergleich indirekt benannten Rolle des Helden und Siegers, das heißt Caesars, und evoziert zweitens, durch das transgressive Erkenntnisinteresse, das sich in dieser Passage in der Wahl der Bilder, der Vergleiche und der Semantik artiku-

29 M. Annaeus Lucanus De bello civili sive Pharsalia (1. Buch, vv. 135s.), cf. Anmerkung 5 in Abélard et Héloïse (2000, 374).

30 Graevenitz (1992, 35ss.) z. B. entwickelt an Abélards anti-augustinischer Stoßrichtung sein theologisches «Negativitätsdenken», zu Augustinus und Lucan: cf. Vinken (2002, 186ss.). 
liert, den ersten Bruderkrieg mit dem berühmten Lehrer für Theologie. Er verdeutlicht damit die Abkehr von der herrschenden Lehrmeinung und demonstriert die Entwicklung des eigenen wissenschaftlichen Ansatzes: seine neuartige Methode des Zweifelns an der dogmatischen Starrheit der Bindung an die Texte und die direkte, von Wissenskompilationen in Form von einfach reproduzierenden Glossen absehende, hermeneutische, textkritische Interpretation, die er im Anschluss durch die Auslegung einer Bibelstelle vorführt. Sie entspricht bereits der dialektisch-rationalen, skeptischen Methode, die er in seiner scholastischen Programmschrift Sic et non formulieren wird (Abélard et Héloïse 2000, 62). ${ }^{31}$

Während Abélards Aufenthalts in Saint-Denis zur Zeit des Konzils von Soissons kommt es zu einem erneuten Bruderzwist, nun mit den Klosterbrüdern. Dabei wird über seine Deutung der Trinität in der ersten Version seines Buches Théologie (Abélard et Héloïse 2000, 79s.) sowie über seinen Verbleib in SaintDenis entschieden, obwohl der eigentliche Streitpunkt und kirchenpolitische Hintergrund eine fehlende Erlaubnis, bei gleichzeitiger Selbstermächtigung, zur Ausübung des Lehramtes ist. ${ }^{32}$ Eine ganze Serie von Naturmetaphern verklammert diese Passagen inhaltlich und legt dabei die poetologische Konstruktion offen: Es geht, metaphorisch gesprochen, darum, den geistigen, von der Zerstörung bedrohten «vigne» (Abélard et Héloïse 2000, 83), den 'Weinstock' des inzwischen universell bekannten Gelehrten weiter gedeihen zu lassen und um die Frage, wo und wie?

Der Bischof Geoffroy de Chartres, der sich in Saint-Denis für die rechtliche Verteidigung Abélards und seiner Schriften einsetzt, gebraucht für Abélards philosophisch universelles, weltumspannendes Wissen zwei biblische Naturvergleiche: ein Zitat des heiligen Hieronymus (einen von Abélards Lieblingsdenkern) vom welt- bzw. ozeanumspannenden Weinstock und ein weiteres von Ovid bzw. Lucan. Dieser Ausspruch greift Ovids Trostwort von den hohen Baumkronen inhaltlich auf und rekurriert erneut, indes unter anderem Vorzeichen, auf die Gegenüberstellung von Standhaftigkeit/Immobilität auf der einen, Zerstörung auf der anderen Seite, die bereits die episch etablierten Baumgleichnisse eröffnet haben:

«Vous savez tous, messeigneurs ici présents, que le savoir universel de cet homme et sa supériorité dans toutes les études auxquelles il s'est attaché lui ont fait de nombreux et

31 Es liegt nahe, hier bereits einen Ansatzpunkt für den spätmittelalterlichen und frühneuzeitlichen Rationalismus sowie für jenen Skeptizismus zu sehen, der die Aufklärung antreiben wird. Darauf verweisen Flasch/Jeck (1997).

32 Cf. zu diesen kirchenrechtlichen Lehramts- und Ausübungsfragen Krautz (Abaelard 1989, 397). 
fidèles partisans; qu'il a, plus que qui que ce soit, étouffé la renommée de ses maîtres et des nôtres, et que sa vigne, si je puis m'exprimer ainsi, a étendu ses rameaux d'une mer à l'autre. Si vous faites peser sur lui le poids d'une condamnation sans l'avoir entendu, ce que je ne pense pas, sa condamnation, fût-elle juste, blessera bien des gens, et il s'en trouvera plus d'un qui voudra prendre sa défense, surtout quand nous ne voyons, dans l'écrit incriminé, rien qui ressemble à une attaque ouverte. On dira, selon le mot de saint Jérôme: «La force qui se montre attire les jaloux, de même que les hautes cimes appellent la foudre»» (Abélard et Héloïse 2000, 83).

Doch das Konzil endet, trotz der prominenten Fürsprache, nach kurzer Klosterhaft mit der Flucht Abélards und dem Rückzug des Philosophen in die Natur mit der anschließenden Gründung des Klosters Paraklet. Beides wird über konventionelle Naturmetaphern artikuliert: den Rückzug in die Wildnis, die alttestamentliche Prophetenaskese und den Verweis auf die antike, philosophische Tradition der Anachorese, die Jünger und Anhänger nach sich zieht: ${ }^{33}$

«Ma retraite ne fut pas plus tôt connue, que les disciples arrivèrent de toutes parts, abandonnant villes et châteaux pour habiter un désert, quittant de vastes demeures pour de petites cabanes qu'ils se construisaient de leurs mains, des mets délicats pour des herbes sauvages et un pain grossier, des lits moelleux pour le chaume et la paille, leurs tables pour des mottes de terre» (Abélard et Héloïse 2000, 91).

Damit sind jene Naturkonzepte aufgerufen, die Abélards eigenes communitasModell fundieren werden. Zugleich wird - in Form einer ganzen Reihe von Anti-

33 So die Textstelle, die neben dem Rückzug in die Natur, im Einklang mit der frühscholastischen Sexualethik, auch auf die Disziplinierung der Leidenschaften zielt: «Telles sont les raisons qui déterminèrent nombre de philosophes à s'éloigner des villes peuplées et des jardins de plaisance où se trouvaient réunis la fraîcheur des campagnes, le feuillage des arbres, le ramage des oiseaux, le cristal des sources, le murmure des ruisseaux, tout ce qui peut charmer les oreilles et les yeux; ils craignaient qu'au milieu de la profusion du luxe et de l'abondance, la vigueur de leur âme ne fût énervée, sa pureté souillée. Et, en effet, il est inutile de voir souvent les choses qui peuvent séduire et de s'exposer à la tentation de celles dont on ne pourrait plus se passer sans peine: voilà pourquoi les Pythagoriciens, évitant tout ce qui pouvait flatter les sens, vivaient dans la solitude et les déserts. Platon lui-même, qui était riche et dont Diogène foulait un jour le lit sous ses pieds souillés de boue, Platon, afin de pouvoir se livrer tout entier à la philosophie, choisit, pour siège de son académie, une campagne déserte et même pestilentielle, loin de la ville, afin que la perpétuelle préoccupation des soins nécessités par la maladie brisât la fougue des passions, et que ses disciples ne connussent d'autres jouissances que celles qu'ils tireraient de l'étude. Tel fut aussi, dit-on, le genre de vie des fils des prophètes, adeptes d'Elisée. Jérôme, qui parle d'eux comme des moines de ce temps, dit entre autres choses: «Les fils des prophètes, que l’Ancien Testament nous représente comme des moines, se bâtissaient de petites cabanes vers le cours du Jourdain, et abandonnaient la foule des villes, pour aller vivre de bouillie et d'herbes sauvages»» (Abélard et Héloïse 2000, 92). 
thesen - die Reflexion zu einem grundlegenden Wertewandel zum Ausdruck gebracht. Wenn sich in der Klostergründung überdies die Verweigerung formuliert, externe Geldspenden anzunehmen, kann man das parallel lesen zu jener weltlich-ökonomischen Öffnung der zeitgenössischen Reformorden, die Abélard, der streng der benediktinischen Regel folgt, ${ }^{34}$ ablehnte, Bernhard von Clairvaux mit seinem Zisterzienserorden hingegen unterstützte. ${ }^{35}$ Der Text stellt die sich anschließende Klostergründung daher in die biblische Tradition der Neugründungen qua Landnahme (etwa der Erzväter) und artikuliert ein auf freundschaftlichem Austausch und Gemeinschaft basierendes Lehr- und Ökonomiekonzept: Gegen die Lehre seines Lehrers Anselm von Laon, die sich gerade dadurch charakterisierte, dass sie kein solides Holz war, setzt Abélard nun sein eigenes, aus solidem Holz gefertigtes Haus und Lehrgebäude:

«À ce moment, ce fut l'excès de la pauvreté qui me détermina à ouvrir une école: ‘Je n’avais pas la force de labourer la terre et je rougissais de mendier . Ayant donc recours à l'art que je connaissais, pour remplacer le travail des mains, je dus faire office de ma langue. De leur côté, mes disciples pourvoyaient d'eux-mêmes à tout ce qui m'était nécessaire: nourriture, vêtements, culture des champs, constructions, si bien qu'aucun soin domestique ne me distrayait de l'étude. Mais, comme notre oratoire ne pouvait contenir qu'un petit nombre d'entre eux, ils se trouvèrent dans la nécessité de l'agrandir, et ils le rebâtirent d'une manière plus solide, en pierres et en bois.

Fondé d'abord au nom de la Sainte-Trinité, placé ensuite sous son invocation, il fut appelé Paraclet, en mémoire de ce que j’y étais venu en fugitif, et qu'au milieu de mon désespoir j’y avais trouvé quelque repos dans les consolations de la grâce divine» (Abélard et Héloïse 2000, 93s.).

Die Naturmetaphorik markiert in der Historia Calamitatum nahezu alle Meilensteine. Dabei sticht die Dominanz der Baumgleichnisse, sowohl als Zitate der antiken Literatur wie der christlichen Tradition, heraus: An ihnen artikuliert sich Abélards Erkenntnisdrang und die Behauptung der eigenen Methode, die er gegen den institutionellen und textexegetischen Dogmatismus einwendet. Strategisch platziert fungieren die eröffneten Analogien als Kommentar auf diesen, dienen geradezu als Kontrastfolie, vor der sich die triumphale institutionelle und methodische Neugründung abheben lässt. Vor diesem Hintergrund sind nun die Naturreferenzen in den Briefen zwei und sechs bis acht $\mathrm{zu}$ betrachten. Diese greifen die Idee und die Metaphern der in der Historia Calamitatum angedachten

34 Cf. Abélard et Héloïse (2000, 168ss.) die Briefe sechs bis acht. Héloïse bittet Abélard zu Beginn des sechsten Briefs, die Regula Benedicti, die sich an den Männerklöstern orientiert, speziell für die Frauenklöster umzuschreiben (Abélard et Héloïse 2000, 169).

35 Cf. hierzu Krautz (Abaelard 1989, 403-405) sowie das Kapitel zum Klosterleben in Hausmann (1996, 102-106). 
«nouvelle plantation» wieder auf. Allerdings, die Natur steht nun im religiösen und institutionellen Kontext der Klosterreform. Es ist zu zeigen, inwiefern sie einem alternativen Gemeinschaftsmodell Kontur verleiht.

\section{Konzept und Begründung einer «natürlichen» Gemeinschaft. Abélards communitas-Modell gemäß eines «ordre naturel»}

«Elle est donc à toi, oui, bien à toi, cette plantation nouvelle dans le saint dessein» (Abélard et Héloïse 2000, 114) - die bekannteste und häufig zitierte Referenzstelle der Natur in den Briefen von Héloïse und Abélard stellt eine kurze, aber sehr exponierte Stelle im zweiten Brief dar. Sie reflektiert auf die Klostergründung und enthält die Aufforderung zur Klosterreform. Doch in der Regel wird sie nicht auf die Verwendung der hier artikulierten Naturkonzepte hin beleuchtet, obschon in ihnen die Gründungs- und Reformanliegen genau genommen begründet werden:

«Après Dieu, tu es le seul fondateur de cet asile, le seul architecte de cet oratoire, le seul créateur de cette congrégation. Tu n'as point bâti sur un fondement étranger. Tout ce qui existe ici est ta création. [...] Pour l'édifier, tu n'as rien emprunté aux richesses des rois et des princes dont tu aurais pu obtenir le concours le plus large et le plus puissant; tu as voulu que rien de ce qui se ferait ne pût être attribué qu'à toi seul. Ce sont les élèves et les écoliers qui, s'empressant à l'envi à tes leçons, te fournissaient toutes les ressources nécessaires; ceux-là mêmes qui vivaient des bénéfices de l'Église et qui ne savaient guère que recevoir des offrandes et non en faire, ceux qui jusqu'alors n'avaient eu des mains que pour prendre, non pour donner, devenaient pour toi prodigues et t'accablaient de leurs libéralités.

Elle est donc à toi, oui, bien à toi, cette plantation nouvelle dans le saint dessein, cette plantation toute remplie de jeunes rejetons, qui, pour profiter, demandent à être arrosés. Par la nature même de son sexe, elle est faible et fragile; ne fût-elle pas nouvelle, à ce titre seul, elle serait faible: aussi exige-t-elle une culture plus attentive et plus assidue, selon la parole de l'apôtre: 〈J'ai planté, Apollos a arrosé; mais c'est Dieu qui a donné l'accroissement〉. L'apôtre, par les enseignements de sa prédication, avait planté et établi dans la foi les Corinthiens auxquels il écrivait; Apollos, son disciple, les avait ensuite arrosés par ses saintes exhortations, et c'est alors que la grâce divine avait donné à leurs vertus de croître» (Abélard et Héloïse 2000, 114s.).

Freilich, in der Naturmetaphorik verbinden sich hier, wie an zahlreichen nachfolgenden Stellen, Klostergründung und Liebesbeziehung. Die Naturreferenzen beziehen sich auf die Liebe zwischen Héloïse und Abélard, insofern sie die beiden durch die Abélard gewidmete, weibliche «nouvelle plantation» zusammenbringen (Abélard et Héloïse 2000, 114). Letztere dient zugleich als Gründungsmeta- 
pher für die sublimierte Überwindung und produktive Übertragung ihrer weltlichen Liebe: Diese wird - unter Rekurs auf eine sehr eigene und etwas eigenwillige Hohelied-Deutung Abélards - konvertiert in das geistige Band des Abtes zur Äbtissin bzw., anders gesagt, des Klerikers Abélard zur Braut Christi, Héloïse. ${ }^{36}$ In der Logik der Zeichen und im Einklang mit der Rhetoriktheorie kann diese Pflanzenmetaphorik als eine metaphorische «oikonomia» verstanden werden, als eine Bewegung von einem Platz an einen anderen. ${ }^{37}$

Die Natursemantik, die im Anschluss in Form von Gleichnissen, Vergleichen, fortgesetzten Metaphern und Zitaten entfaltet wird, verdichtet jedoch noch mehr: Sie verdeutlicht zunächst, dass die neue Ordnung in einer neuen Institution verwirklicht werden muss. In einem ganzen Netz von Oppositionen werden Klostergründung und -reform («nouvelle plantation») daher dem «vigne», der Lehren und Disziplin Abélards gegenübergestellt:

«C'est vainement que tu cultives cette vigne que tu n'as pas plantée de ta main, et dont «la douceur a tourné pour toi en amertume;; tes admonitions incessantes sont stériles, tes sacrés entretiens inutiles: songe à ce que tu dois à la tienne, au lieu de consacrer ainsi tes soins à celle d'autrui. Tu enseignes, tu prêches des rebelles: peine perdue. C'est en vain que tu sèmes devant des pourceaux les perles de la parole divine; tu te prodigues à des âmes endurcies. Considère plutôt ce que tu dois à des cœurs dociles. Tu te donnes à des ennemis; pense à ce que tu dois à tes filles. Et sans parler des autres, pèse le poids de la dette que tu as contractée envers moi. Peut-être mettras-tu plus de zèle à t'acquitter vis-à-vis de toutes ces femmes qui se sont données à Dieu dans la personne de celle qui s'est donnée exclusivement à toi» (Abélard et Héloïse 2000, 114s.).

Diese Gegensatzpaare ordnen der «nouvelle plantation» die positiven Aspekte, dem «vergeblich kultivierten Weinstock» die negative Seite zu: Ist erstere eine vollkommen eigene Schöpfung («seul fondateur», «seul architecte», «seul créateur»), die des Anbaus und der Fürsorge, der Pflege, der «culture» im eigentlichen und umfassenden Sinn sowie der "grâce divine» bedarf, um all die jungen Knospen zum Wachsen und Blühen zu bringen, muss der Weinstock, «pas plantée de ta main», als «stérile» gelten. Seine Kultivierung durch die «parole divine» ist vergeblich, «peine perdue». Alle Naturbilder markieren den Abschied von der alten Ordnung auf der einen, die Begründung einer neuen Ordnung auf der anderen Seite.

36 Den Liebeskonventionen der Zeit entsprechend, werden Héloïse und Abélard als Beispiel für eine Überwindung und Sublimierung der leidenschaftlichen Liebe gelesen, cf. einführend: Hausmann (1996, 187s.), ferner Barbara Vinken (2002). Zur Theologie der Liebe bei Abélard unter Berücksichtigung des Gesamtwerks und der Liebe (caritas) als Teil des mittelalterlichen Tugendverständnisses, Podlech (1990).

37 Zum Beispiel Parker (1998, 312-331). 
Dass bereits diese Passage grundiert ist von Fragen der Gouvernementalität, die Abélard in der Folge im Rahmen seiner Klosterregeln für die Nonnen beschäftigen werden, ist weit mehr als ein Nebenaspekt (der nicht erst heute, sondern bereits für die Rezeptionsgeschichte des Briefwechsels so uninteressant erschienen sein muss, dass er kaum eine Rolle gespielt hat oder gar nicht erst mitpubliziert wird). Dabei artikuliert sich Abélards communitas-Modell, das ich als Konzept einer «natürlichen» Gemeinschaft fassen möchte, hier am deutlichsten: zum einen, weil es über die Metaphorik der Ordnung der Natur artikuliert wird und zudem über ein gegenseitiges, natürliches, freundschaftliches Geben und Nehmen Gestalt gewinnt, zum anderen, da auf diese Weise eine gewisse Parallelität zu seinen Modellen des «natürlichen Rechts» und der «natürlichen Religion» entsteht. ${ }^{38}$

Es ist daher lohnenswert, die Rede vom Geben und Nehmen der Schüler Abélards, Héloïses Apostel-Zitat und Blumenmetaphorik mit Blick auf ein (Abélard'sches) communitas-Konzept zu betrachten. Denn in den späteren Ratschlägen an die Äbtissin Héloïse, ab dem achten Brief, wird sich Abélard am Beispiel des Klosterbetriebs eingehend zu Fragen der Ökonomie äußern, zu Gesetzen und Ordnung des 'Hauses', wie er es getreu der Etymologie von «oikonomia» (aus griech. oikos und nomos) nennt. ${ }^{39}$ Auch deutet sich in diesem Kontext die Spannung von jenen zwei dichotomen Gouvernementalitätsparadigmen an, die sich mit Georgio Agamben als politisch-theologisch vs. ökonomisch bezeichnen lassen..$^{40}$ Abélard benennt sie am Ende des siebten Briefs, wenn es im Anschluss an seine Ausführungen zur «Herrschaft» («relative à l'autorité de votre ordre») heißt: «vous embrasserez maintenant les devoirs auxquels vos vœux vous obligent avec d'autant plus de zèle que vous en connaissez mieux l'excellence» (Abélard et Héloïse 2000, 247). Doch gerade weil sich Agamben für die Formierung und Formulierung dieser beiden Paradigmen auf andere historische Zeugnisse stützt und gerade weil Abélards Position, die sich auf eine davon grundverschiedene, antike Traditionslinie beruft bzw. diese gewissermaßen gegen die theologische führt, die antike in den christlichen Kontext seines Klosters einbringt, weitgehend ohne Wirkung geblieben $\mathrm{zu}$ sein scheint, erscheint es mir interessant, seine Konzeption in den Blick zu nehmen.

Die Metaphorik der Pflanzen und vor allen Dingen der Rekurs auf den «ordre naturel» der Geschlechter bzw. einer der Schöpfung entsprechenden weiblich/ männlichen «nature» (v.a. im zweiten und achten Brief), auf welche die Argu-

38 Zu Abélards Naturrechtkonzept: Marenbon (1992, 216-218), zur «natürlichen Religion»: Flasch (2000, 252).

39 Die Passage findet sich in der deutschen Ausgabe (Abaelard 1989, 244s.).

40 Cf. zu diesen beiden Paradigmen Agamben (2010, 11-18). 
mentation zur Klostergründung und Klosterordnung mehrfach rekurriert, kann an dieser Stelle als zentral angesehen werden. ${ }^{41}$ Und es zeigt, dass es sich hier nicht nur um eine für die Geschlechterverhältnisse und die topische Orientierung an der weiblichen «nature» (Schönheit) maßgebliche Metaphorik handelt, sondern auch und vor allem um eine Reflexionsfigur, durch die sich die Ausführungen zur Klosterreform und zu ihrer Ökonomie denken lassen. Was über die Beziehung der Geschlechter gegen Bernard von Clairvaux eingewendet wird, der die Klosterordnung auf der Grundlage der Hoheliedmystik auf den überbrachten Geschlechterverhältnissen beruhen lässt und das männliche Verhältnis des Abts zu seinen Mönchen als ein weibliches beschreibt, ${ }^{42}$ kann - auf der Basis des zugrundeliegenden Naturkonzepts gelesen - in den Kontext grundlegender ökonomischer Paradigmen eingerückt werden.

\section{Fazit}

Der Text lässt keinen Zweifel, dass sich Abélards Konzeption einer «natürlichen» Gemeinschaft gegen die zunehmende weltliche Öffnung der Klöster richtet, die, wie die Zisterzienser, Schenkungen und weltlich-ökonomischen Interessen kaum ablehnend gegenüberstehen. ${ }^{43}$ Zur Zeit des allmählichen Verschwindens der feudal-aristokratischen Wertewelt und im Kontext eines zivilisatorischen Wandels durch das zunehmende Anwachsen der Städte, ${ }^{44}$ kann man sagen, setzt und schafft Abélard insbesondere, aber nicht erst, in den Briefpassagen zur Klosterordnung ein Exemplum einer «natürlichen» Gemeinschaft. Explizit spielt er in den autobiografischen Passagen zum eigenen Werdegang, dann zur Klostergründung und -wirtschaft auf den zivilisatorischen Wertewandel der Zeit an, der mit der zunehmenden Verstädterung korreliert. «Si [...] tu désires être moine, c'est-àdire solitaire, que fais-tu dans les villes, qui certes ne sont pas la demeure des solitaires, mais celle de la foule? Chaque établissement a ses modèles» (Abélard et Héloïse 2000, 259) ${ }^{45}$ - Abélards Ökonomie des Klosters und der Klosterregeln

41 Abélard, das wird an dem Rekurs auf den «ordre naturel» deutlich, greift eine andere, stoische, naturrechtliche Tradition auf, der zufolge - so kann man vielleicht mit Hartung (1999) argumentieren - die «Naturordnung die Grundregeln gerechten Handelns, das zudem verbindlich ist, d. h. eine Pflicht (officium) impliziert», enthält, cf. Hartung (1999, 51).

42 Zum Streit von Abélard mit Bernhard von Clairvaux, cf. allgemein Rieger (2002) sowie mit Blick auf die Deutung der Geschlechterverhältnisse, Vinken $(2002,193)$.

43 Cf. z.B. Hausmann (1996, 102-106).

$44 \mathrm{Zu}$ diesen realhistorischen Hintergründen: Flasch (2002, 237).

45 Diese Argumentation setzt sich einige Seiten lang fort und dient der Begründung des zivilisationsfernen Klosterstandorts jenseits der ständigen städtischen Verführungen. 
ist deswegen eine vorbildliche, das Gegenteil der Menge/Stadt und doch reflektiert sie auf die weltliche Ordnung, setzt sich zu dieser - als eine exemplarische ausdrücklich ins Verhältnis.

Man darf vor diesem Hintergrund darauf schließen, dass die Konturierung eines communitas-Modells als eine Reaktion auf die realen Bedürfnisse und Verhältnisse der Zeit zu verstehen ist. Und doch: Ist das, was sich so leicht als soziokultureller Kontext lesen lässt, nicht zugleich auch ein ziemlich deutlicher Verweis auf jenes aristotelische Gegensatzpaar Stadt ('polis') und Haus ('oikos'), das Aristoteles' an der Verwaltung des Hauses orientierten Ökonomie zugrunde liegt? ${ }^{46}$ Damit wäre Abélards Kloster- als Hausordnung zu verstehen, die im Gegensatz zur von außen und einer Vielheit regierten Stadt und gegen die ebenfalls von außen, vom Kirchenstaat und unzähligen Diözesen verwalteten und, schlimmer noch, von außenorientierter Herrlichkeit bestimmten brudermörderischen Klostergemeinschaften - eine Ökonomie aufzeigt, die von innen heraus verwaltet wird, einem einheitlichen Prinzip folgt. Das würde auch noch einen weiteren Rekurs auf Aristoteles erklären. Verweisen bereits die Bemerkungen zu einem gegenseitigen Geben und Nehmen im Rahmen der Klostergründung darauf, dass die Klosterökonomie von einem antiken (bzw. urchristlichen) Freundschaftskonzept getragen ist, bestätigt dies erst recht ein Blick auf den neuen affektiven Bund des einstigen leidenschaftlichen Liebespaars: Nicht (nur) eine sublimierte und in einen neuen Bund konvertierte Liebe, sondern vor allem der Ersatz der leidenschaftlichen Liebe durch ein Freundschafts- und Fürsorgekonzept macht diesen neuen Bund wie, in Erweiterung, die ganze Klostergemeinschaft aus. Könnte es sein, dass sich hier bereits jene conversio des antiken, aristotelischen Freundschaftsbegriffs zu dem christlichen Konzept der Fürsorge, Nächstenliebe, caritas ankündigt, welche das scholastische Freundschaftskonzept ausmacht und das man in der Regel (erst) bei Thomas von Aquin ansetzt? ${ }^{47}$ Ausdrücklich appelliert Héloïse, gleich zu Beginn des zweiten Briefs, an Abélards Fürsorgeverpflichtung, an seine «amitié» und «confraternité», die er in seinem Trostbrief zwar dem Freund, nicht aber seinen eigentlichen «amies», ihr und den

46 Aristoteles (1989, 1261a).

47 Cf. Thomas von Aquin, Summa theologica, 2-2, qu. 23, a 1c bzw. alle 8 Artikel der questio 23 zur «caritas» im zweiten Teil des zweiten Teils, der den theologischen Tugenden gewidmet ist, lateinisch nach: <http://www.corpusthomisticum.org/sth3023.html> [letzter Zugriff: 9.7.2015] sowie nach der englischen Übersetzung: <http://www.sacred-texts.com/chr/aquinas/summa/su m278.htm> [letzter Zugriff: 9.7.2015]. Exemplarisch zu dieser Frage der «caritas est amicitia», Kablitz (1993, 164). Abélard war, soweit man weiß, nicht das gesamte Werk des Aristoteles zugänglich; Aristoteles Nikomachische Ethik allerdings, die das auf ähnlichen Sitten beruhende Freundschaftskonzept (zwischen Mann und Frau) enthält, kannte er. Cf. zu diesem Konzept, Aristoteles (1986, 8. Buch). 
Nonnen angedeihen ließe (Abélard et Héloïse 2000, 113). Daneben erscheint sein Konzept einer «natürlichen» Gemeinschaft in seiner Interpretation des Hohelieds im fünften Brief besonders deutlich greifbar. In den mannigfach zitierten Stellen zur Installation des neuen Liebesbundes am Beispiel des Hohelieds läuft die conversio des Bundes zum Gemeinschaftsmodell bereits mit. Abélards Deutung (insbesondere der sogenannten Schwarzen) kann deshalb mit Blick auf das neue Gemeinschaftsmodell gelesen werden, das seiner eigenen Klostergründung in praxi zu Grunde liegt, z. B.:

«Cette noirceur même des tribulations corporelles arrache aisément le cœur des fidèles à l'amour des choses terrestres, pour les attacher aux désirs de l'éternelle vie; souvent elle les enlève à la tumultueuse agitation de la vie du siècle et les pousse vers les mystères de la vie contemplative. C'est ainsi que, selon le bienheureux Jérôme, Paul embrassa le premier notre genre de vie, je veux dire la vie monacale.

Ces vêtements grossiers aussi sont plutôt faits pour la retraite que pour le monde; ils sont proprement en harmonie avec la pauvreté et la solitude qui conviennent au caractère de nos vœux. Car rien n'excite plus vivement à se produire en public que le luxe de la toilette, luxe qu'on ne recherche qu'en vue des pompes de ce monde et d'une vaine gloire» (Abélard et Héloïse 2000, 148).

Es wundert daher nicht, wenn sich in Abélards Hohelied-Interpretation die Argumentationslinie einer natürlich-freundschaftlichen, weltlich-organisatorischen Brüder- und Schwesterlichkeit angekündigt findet, die sich in seinen Begründungen zum Doppelkloster im achten Brief fortsetzt und in der Adaption der, ausschließlich männlichen, benediktinischen Klosterregeln für die Nonnen ihren schriftlichen Ausdruck findet. ${ }^{48}$ Abélards Transformationen der mystisch-christlichen Hohelied-Deutung lösten bei Bernhard von Clairvaux, der das Mysterium mystischer Gottesliebe bedroht sah, bekanntlich größte Empörung aus und führten letztlich zur Verurteilung Abélards als Ketzer. Sie gehen über die «geläufigen Analogien» hinaus, stehen dem jenseitsorientierten Hohelied-Verständnis seines Widersachers Bernhard von Clairvaux diametral entgegen. ${ }^{49}$ Sie sind allerdings nicht radikal in einem modernen Sinne der Dekonstruktion der allegorischen Hohelied-Auslegung. Sie sind radikal in dem Sinne der weltlichen Anwendbarkeit, der Transformation in die weltlichen Bereiche des Klosterlebens, der Übertragung des Bundes mit dem Einen auf die gesamte Einheit und Organisation des Klosterlebens.

48 Vor dem Hintergrund des antiken, aristotelischen Freundschaftskonzepts betrachtet, spricht einiges dafür, dass Héloïse Bitte um die Angleichung der etablierten Klosterregeln für Nonnen Teil einer solchen, auf Ähnlichkeit der Sitten basierenden, Freundschaftskonzeption ist.

49 Krautz (Abaelard 1989, 414). 
Sie erschließen damit dem Hohelied einen neuen Deutungshorizont. Mit seiner weltlichen Übertragbarkeit gründet Abélard - im gemeinschaftlichen Briefwechsel - eine Gemeinschaft in Gott und rekurriert zugleich auf ein stoisches, antikes Freundschaftskonzept, das in diesen Bund konvertiert wird, legt dem neuen «ordre naturel» eine antike Genealogie zugrunde, welche die Klosterökonomie ebenso wie die Geistesgemeinschaft trägt.

\section{Bibliografie}

Abaelard, Der Briefwechsel mit Heloisa, ed. Krautz, Hans-Wolfgang, Stuttgart, Reclam, 1989. Abaelard, Peter, Scito te ipsum [Ethica] Erkenne Dich selbst, ed. und übers. Steger, Philipp, lateinisch-deutsch, Hamburg, Felix Meiner, 2006.

Abélard et Héloïse, Correspondance, edd. Gilson, Étienne/Bouyé, Édouard, Paris, Gallimard, 2000.

Achtner, Wolfgang, Vom Erkennen zu Handeln. Die Dynamisierung von Mensch und Natur im ausgehenden Mittelalter, Göttingen, Vandenhoeck \& Ruprecht, 2008.

Agamben, Giorgio, Herrschaft und Herrlichkeit. Zur theologischen Genealogie von Ökonomie und Regierung. Homo sacer Il.2, übers. Hiepko, Andreas, Frankfurt am Main, Suhrkamp, 2010.

Aristoteles, Nikomachische Ethik, ed. und übers. Dirlmeier, Franz/Schmidt, Ernst A., Stuttgart, Reclam, 1986.

Aristoteles, Politik. Schriften zur Staatstheorie, ed. und übers. Schwarz, Franz F., Stuttgart, Reclam 1989.

Asper, Markus, Leidenschaften und ihre Leser. Abaelard, Heloise und die Rezeptionsforschung, in: Hasse, Dag Nikolaus (ed.), Abaelards «Historia calamitatum». Text - Übersetzung literaturwissenschaftliche Modellanalysen, Berlin et al., De Gruyter, 2002, 105-139.

Baumgarten, Alexander Gottlieb, Meditationes philosophicae de nonnullis ad poema pertinentibus / Philosophische Betrachtungen über einige Bedingungen des Gedichtes, übers. Paetzold, Heinz, Hamburg, Meiner, 1983.

Benton, John F./Prosperetti Ercoli, Fiorelle, The Style of «Historia Calamitatum», Viator 6 (1975), 59-86.

Campe, Rüdiger/Menke, Christoph/Haverkamp, Anselm, Baumgarten-Studien. Zur Genealogie der Ästhetik, Berlin, August, 2014.

Flasch, Kurt, Das philosophische Denken im Mittelalter. Von Augustin zu Machiavelli, Stuttgart, Reclam, 2000.

Flasch, Kurt/Jeck, Udo Reinhold, Das Licht der Vernunft, München, Beck, 1997.

Graevenitz, Gerhart von, Differenzierung und Differenz. Grundlagen der Autobiographie in Abaelards und Heloises Briefen, in: Janota, Johannes/Sappler, Paul/Schanze, Frieder (edd.), Festschrift Walter Haug und Burghart Wachinger, vol. 1, Tübingen, Niemeyer, 1992, 25-45. Hartung, Gerald, Die Naturrechtsdebatte: Geschichte der Obligatio von 17. bis 20. Jahrhundert, Freiburg im Breisgau/München, Alber, 1999.

Hausmann, Frank Rutger, Französisches Mittelalter, Stuttgart/Weimar, Metzler, 1996.

Jolivet, Jean, Éléments du concept de nature chez Abélard, in: La filosofia della natura nel Medioevo. Atti dei III Congresso internationale di filosofia medioevale, Milano, Vita e Pensiero, 1966, 297-304. 
Kablitz, Andreas, Boccaccios Decameron zwischen Archaik und Modernität. Überlegungen zur achten Novelle des zehnten Tages, in: Kablitz, Andreas/Schulz-Buschhaus, Ulrich (edd.), Literarhistorische Begegnungen. Festschrift zum sechzigsten Geburtstag von Bernhard König, Tübingen, Narr, 1993, 147-182.

Krings, Hermann, Ordo: philosophisch-historische Grundlegung einer abendländischen Idee, Hamburg, Meiner, ${ }^{2} 1982$.

Marenbon, John, Abelards concept of natural law, in: Zimmermann, Albert/Speer, Andreas (edd.), Mensch und Natur im Mittelalter. Veröffentlichungen des Thomas-Instituts der Universität zu Köln, vol. 2, Berlin, De Gruyter, 1992, 609-621.

Marenbon, John, The Philosophy of Peter Abelard, Cambridge, Cambridge University Press, 1997.

Moos, Peter von, Mittelalterforschung und Ideologiekritik. Der Gelehrtenstreit um Héloise, München, Fink, 1974.

Moos, Peter von, Abaelard, in: Flasch, Kurt/Jeck, Udo Reinhold, Das Licht der Vernunft, München, Beck, 1997, 36-45.

Parker, Patricia, Metapher und Katachrese, in: Haverkamp, Anselm (ed.), Die paradoxe Metapher, Frankfurt am Main, Suhrkamp, 1998.

Perkams, Matthias, Liebe als Zentralbegriff der Ethik nach Peter Abaelard, Münster, Aschendorff, 2001.

Podlech, Adalbert, Abaelard und Heloisa oder: Die Theologie der Liebe, München/Zürich, Piper, 1990.

Rieger, Reinhold, Petrus Abaelard. Theologie im Widerstreit, in: Köpf, Ulrich (ed.), Theologen des Mittelalters. Eine Einführung, Darmstadt, Wissenschaftliche Buchgesellschaft, 2002, 61-78.

Schmeidler, Bernhard, Der Briefwechsel zwischen Abaelard und Héloise als eine literarische Fiktion, Zeitschrift für Kirchengeschichte 54 (1935), 323-338.

Vinken, Barbara, Die Autorität der Form in Abaelard und Heloise, Deutsche Vierteljahrsschrift für Literaturwissenschaft und Geistesgeschichte 76 (2002), 181-194.

Wegmann, Milène, Naturwahrnehmung im Mittelalter im Spiegel der lateinischen Historiographie des 12. und 13. Jahrhunderts, Bern, Lang, 2005.

Zimmermann, Albert/Speer, Andreas (edd.), Mensch und Natur im Mittelalter. Veröffentlichungen des Thomas-Instituts der Universität zu Köln, 2 vol., Berlin, De Gruyter, 1992. 